Article

\title{
Integrating Virtual Reality and Digital Twin in Circular Economy Practices: A Laboratory Application Case
}

\author{
Roberto Rocca * ${ }^{\circ}$, Paolo Rosa ${ }^{\circledR}$, Claudio Sassanelli, Luca Fumagalli and Sergio Terzi \\ Department of Management, Economics and Industrial Engineering, Politecnico di Milano, Piazza L. da Vinci 32, \\ 20133 Milan, Italy; paolo1.rosa@polimi.it (P.R.); claudio.sassanelli@polimi.it (C.S.); \\ luca1.fumagalli@polimi.it (L.F.); sergio.terzi@polimi.it (S.T.) \\ * Correspondence: roberto.rocca@polimi.it; Tel.: +39-02-2399-2729
}

Received: 13 December 2019; Accepted: 8 March 2020; Published: 14 March 2020

check for updates

\begin{abstract}
The increasing awareness of customers toward climate change effects, the high demand instability affecting several industrial sectors, and the fast automation and digitalization of production systems are forcing companies to re-think their business strategies and models in view of both the Circular Economy (CE) and Industry 4.0 (I4.0) paradigms. Some studies have already assessed the relations between CE and I4.0, their benefits, and barriers. However, a practical demonstration of their potential impact in real contexts is still lacking. The aim of this paper is to present a laboratory application case showing how I4.0-based technologies can support CE practices by virtually testing a waste from electrical and electronic equipment (WEEE) disassembly plant configuration through a set of dedicated simulation tools. Our results highlight that service-oriented, event-driven processing and information models can support the integration of smart and digital solutions in current $\mathrm{CE}$ practices at the factory level.
\end{abstract}

Keywords: virtual reality; digital twin; circular economy; industry 4.0; disassembly; laboratory application case

\section{Introduction}

Today, manufacturing companies are coping with two types of "instabilities" influencing industrial markets. First, customers are becoming even more aware of the effects of both climate change and the depletion of natural resources. This issue pushed the European Union (EU) and other intranational institutions to define even more restrictive guidelines to be adopted by companies in terms of product and process sustainability. During the last few decades, these guidelines have been translated in the Circular Economy (CE) paradigm, or a synergistic view between ecological and economic systems [1,2]. Second, the intrinsic complexity of current industrial markets (e.g., shorter product lifecycles and higher mass customization) is even more dependent on automated and digital solutions supporting companies for meeting with customer's needs. Trying to include all these digital solutions under the same umbrella, the paradigm of Industry 4.0 (I4.0) has been recently invented, entailing the development of high-tech strategies and internet-based technologies enabling the creation and delivery of added value for organizations and society [3]. Together, CE and I4.0 are forcing companies to re-think their business strategies and models, since both these strategies are complementary, and synergistic effects can be established between them [4]. However, despite many works dealing with the interaction between I4.0 and CE [5], the way in which digital technologies can favor the transition toward CE has been rarely assessed in a real context [6]. Previous studies [4] have looked at various aspects of the $\mathrm{CE}$ and digital transformation, structuring a work frameworks under the principles of the 
$\mathrm{CE}$ and the pillars of sustainability and proposing the development of standardization of the CE as a paradigm for sustainability under the potential offered by digital transformation. The proposed framework is composed by four dimension of interest (i.e., product, process, facilities, and business) and by three levels-(i) CE strategy standards; (ii) CE standards; (iii) CE maturity norms. Starting from this, the present work wants to practically demonstrate how digital technologies can support CE by presenting a laboratory application case. To this aim, attention has been paid to disassembly processes (i.e., process dimension) for triggering better End-of-Life (EoL) strategies for the valuable product component recovery and on the third analysis level (i.e., CE maturity norms), intended as the degree of maturity in the implementation of the $\mathrm{CE}$ in an organization through the incorporation of digital technologies to enable circular industrial metabolism 4.0. Here, I4.0-based technologies support current CE practices by virtually and practically testing a waste from electrical and electronic equipment (WEEE) disassembly plant configuration through a set of dedicated simulation tools and a fully automatized manufacturing line. The paper is organized as follows: Section 2 presents the research methodology adopted within this paper, the adopted I4.0 technologies, and similar laboratory application cases by pointing out the interaction between I4.0 technologies and CE. Section 3 shows the results coming from different tests. Section 4 discusses the obtained results. Finally, Section 5 offers some concluding remarks and potential future research trends.

\section{Materials and Methods}

In 1992, the physicist Robert Frosh [7] introduces the concept of an analogy between natural ecosystems and industrial ecosystems - the "industrial ecology." For the first time, the ecosystem concept was applied to the industrial sphere, connecting the metabolism between industries [8]. Industrial ecology allows researchers to focus on the facility level, on the inter-firm level, and on the regional or global level [9]. Referring to a manufacturing system, the linear model is based on a static view of the flows that characterize the logistics-production chain, which starts from raw materials supplying and processing, up to the finished product disposal. On the other hand, the Circular Economy (CE) systems provide for a more efficient and more effective use of resources, where the flows are not static and bound by one-directionality, but they fall circularly in the upstream phases of the production system [10]. In order to mitigate the damages of linear systems, environmental policies have so far focused their attention on pollution and emissions. Nevertheless, this "end-of-pipe" policy, which is now obsolete, is integrated into a new sustainability perspective that focuses on the exploitation of renewable raw materials and energies to offset the inputs of non-renewables [11]. Eco-efficient techniques try to minimize the volume, speed, and toxicity of material flows but are unable to alter their linear progression. Besides these, the concept of eco-efficacy proposes the transformation of products and related material flows in order to establish a support relationship with ecological systems and future economic growth.

The CE, according to the definition given by Ellen MacArthur Foundation [12], acts with a logic that, in addition to achieving sustainability in the production mechanisms and consumption, provides for the reconstruction of social and natural capital. This concept is combined with change in the behavior of the final consumer. It also provides multiple value creation mechanisms that are decoupled from the consumption of finite resources [12]. For this reason, the "cradle to cradle" philosophy characterizes these systems. In this way, it is possible to extend the final phase of the product life cycle, for example, through a different reuse. It is also possible to stretch the entire cycle by enhancing the efficiency and the efficacy of the individual phases due to the circular flow reintegration. This approach focuses on understanding how resource flows can have a positive impact on the environment, rather than thinking about how to reduce negative impacts. The aim of the circular model is to have integrated processes between technical and biological flows and their interconnected use minimizing or eliminating waste [13]. What is important in the transition from a linear to a circular economy remains the creation of a sustainable system and the ability to capture the value that would normally be lost with linearity, which would then result in an economic loss in the long run. 
As evidenced in Section 1, many works have already assessed the interaction between I4.0 and CE. Trying to put together the sustainability-oriented and technology-oriented views under the same umbrella, the concept of Smart Sustainability has been proposed by experts as a new way for making goods and managing production processes in a more sustainable way by exploiting smart technologies. As pointed out by the authors of reference [5], experts consider four main ways to describe the relation between $\mathrm{I} 4.0$ and the CE:

(i) digitalization of the CE, considering I4.0 technologies as a set of opportunities supporting enterprises in increasing their circular degree;

(ii) the role that I4.0 technologies have in enabling circular business models related to the stakeholder's involvement (e.g., customers);

(iii) other CE-related aspects (e.g., resource efficiency and lifecycle management), where I4.0 technologies are enablers of innovative ways for monitoring and optimizing resources performances;

(iv) disassembly and supply chain management with I4.0 technologies as element for developing and managing supplier-customer relationships.

Similarly, the integration of smart and digital (I4.0-based) technologies in manufacturing plants inspired the definition of new terms such as "Smart Manufacturing" and-within company boundaries- "Smart Factory" [3]. Together, all these technologies are changing the classic approach of industrial processes supporting the storage, transportation, and transformation of raw materials into useful products. Therefore, the International Standards of Automation set of standards (ISA-95) [14]—containing all the commonly agreed definitions of the automation pyramid's layers-must also evolve (see Figure 1, below). However, this evolution through I4.0 technologies can maintain the old pyramid intact by acting on the ways different layers can interact, gather information from the field, and store data [15-17]. This is possible thanks to the Cyber Physical Systems (CPSs) connection among the layers, allowing a more flexible pyramid exploitation.

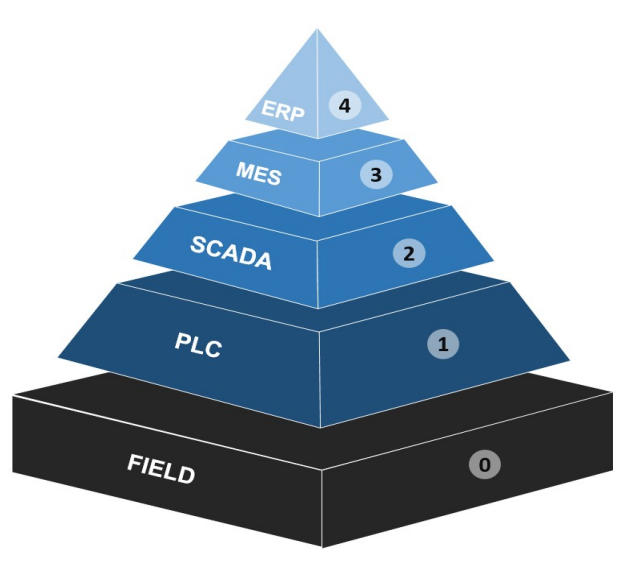

(a)

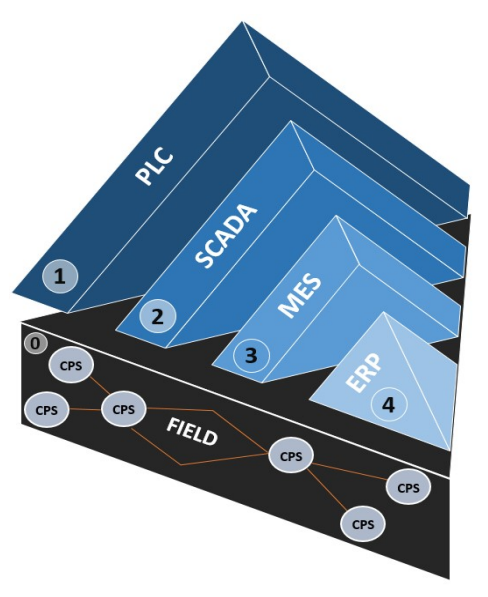

(b)

Figure 1. ISA-95 automation pyramid (a) and flexible automation pyramid (b).

The enabling 4.0 technologies exploited in the laboratory application case presented in this work are described below. Subsequently, the detailed description of the experiment that has been developed is described, presenting some application results with the scope to practically demonstrate within a laboratory pilot plant the I4.0 technologies exploitation for the digitalization of the disassembly processes. The first application result is related to the monitoring and optimization of energy performance, while the second regards the integration between the MES software and the facility, closing the information flow between the physical and digital parts of the system. 


\subsection{Enabling Technologies}

Considering these technological tendencies, it can be argued that a knowledge gap exists in terms of how organizations can develop integrated smart and sustainable operations in view of a more general I4.0-supported CE strategy. Fortunately, the advent of I4.0 technologies provided immense opportunities for unlocking the potential of CE practices through a higher level of connectivity and efficiency [18]. The following sub-sections describe in detail all the I4.0 technologies and techniques adopted within this paper. The technologies described below have been chosen for their potential in the field of the experiment presented in this work. Moreover, they are the most suitable among those present in Industry 4.0 Lab of Politecnico di Milano, where the experiment was conducted.

\subsubsection{Cyber Physical Systems}

Cyber Physical Systems (CPSs) are ICTs systems [15] integrating computation, networking, and physical processes [19-21]. They represent the key object linking together all I4.0 technologies, by enabling a real improvement of goods and services. CPSs integrate a cyber space with physical processes and objects by connecting machine tools and devices as a network, thus monitoring and exchanging real-time data for decision making. They represents a new way to enable either better lifecycle management of products and services (e.g., for maintenance reasons) or to optimize remanufacturing practices or multi-agent systems for managing the extraction of natural resources [5]. This way, resources (products, material, energy) can be allocated efficiently based on intelligent cross-linked value creation modules [22]. Together with the Internet of Things (IoT) and Cloud Computing (CC), CPSs can inform users either about components and materials embedded into products or disassembly and recycling procedures [23] in order to enable a more efficient reintroduction into new product value chains.

\subsubsection{Internet of Things}

Internet of Things (IoT) is the digital technology that can be most easily integrated in CE practices $[18,24,25]$. Its role lies in collecting data from the field and then transferring that data (for example, through CPS [3]) to other I4.0 technologies (e.g., Big Data \& Analytics (BDA) and CC) tools that are able to analyze them and extrapolate information for better decision-making at either the product or process level [18,26-28]. Under this perspective, IoT may intrinsically increase resource efficiency, extend product lifespan, and close the loop [24]. By collecting and analyzing product-related data through IoT, they can be redesigned, maintained, upgraded, disassembled, or recycled more easily $[25,29]$. In addition, IoT also provides process-related data by optimizing remanufacturing and recycling practices and enabling better production planning and control [24,28]. For these reasons, IoT adoption in CE practices can enable (i) new waste management strategies, (ii) improvement of the processes circularity level, and (iii) creation of smart industrial environments or dynamic feedback control loops $[5,30,31]$.

\subsubsection{Simulation}

Within the I4.0 paradigm, simulation is used to replicate real world behaviours in virtual environments. This way, physical and virtual dimensions coexist and are synchronized in real-time [19]. However, synchronization requires full data models of alternative scenarios to be simulated [32-34]. This issue led to the concept of Digital Twin (DT), or a virtual representation of physical objects coping their behaviour through a real-time data acquisition from the field [16,35]. A DT not only allows a prognostic assessment at design stage (static perspective), but also a real-time synchronization and optimization of the virtual object (dynamic perspective) [36]. From a CE perspective, simulation is logically related to either better management of complex supply chains (e.g., closed-loop chains through disassembly process optimization within EoL phase) or the remanufacturing of complex products [5]. Even if DT could be easily adaptable to CE practices, only a few papers have dealt with 
this topic. For example, Wang et al. [37] described the idea of adopting a DT in different CE practices, either for the virtual optimization of disassembly (e.g., monitoring materials and energy consumptions) or recycling and remanufacturing processes (e.g., storing knowledge about components and materials embedded into products). Another example of simulation is based on the concept of Augmented Reality (AR) and Virtual Reality (VR). Considering what was reported by the authors of reference [29], AR/VR could represent a valuable element for improving disassembly and remanufacturing processes.

\subsubsection{Autonomous Robots}

The last I4.0 solution evaluated in this literature review is represented by autonomous robots. For many decades, robots have been used in manufacturing processes for complex assignments. However, the latest developments in robotics have made them even more autonomous, flexible, and cooperative. The disassembly of products is a key process in the treatment of WEEE. When performed efficiently, it enables the maximization of resources re-usage and a minimization of pollution. Despite this, currently employed automation solutions are mainly custom-oriented and not quite suited to cope with the dynamic nature of the disassembly environment resulting from the wide variety of products to be disassembled as well as their general shape at their disposal (e.g., scratches and fractions) [38]. Within the I4.0 paradigm, collaborative robots (co-bots) can safely interact with humans and learn from them. This flexibility makes them suitable for supporting current CE practices, especially during disassembly and remanufacturing operations [39].

\subsubsection{Generic Application Cases: I4.0 Technologies Supporting CE Practices}

The literature reports some examples of generic application cases linking I4.0 technologies with current CE practices, especially for remanufacturing, maintenance, and disassembly (ordered by number of works). Considering remanufacturing and disassembly processes, some examples are available. Yang et al. [18] presented two case studies showing how I4.0 technologies can increase performance in remanufacturing processes. A smart remanufacturing cell (focused on repairing activities) of Computer Numerical Control (CNC) machine tools has been simulated by gathering data from the field through smart sensors. A CPS was thus developed for the real-time monitoring of CNC machines and the definition of maintenance activities. French et al. [40] described the use of robots, BDA, and IoT for improving remanufacturing performances in the aerospace industry. By incorporating machine vision systems for characterization, inspection, and fault detection, alongside advanced real-time sensor data acquisition for monitoring and evaluating the welding process, manual remanufacturing can become a smart process.

Other experts focus more on the usage phase of products, especially on maintenance activities. Barbosa et al. [41] show how CPSs can be integrated into products via two industrial cases (trains and washing machines manufacturing), covering production, use, and maintenance lifecycle phases. In the first case, CPSs were used for monitoring, gathering data from different lifecycle stages and improve the capitalized knowledge on products. In the second case, CPSs were adopted to improve both production efficiency and product quality through constant monitoring and optimization of the production processes. Lee et al. [19] described the exploitation of CPSs and simulation tools for developing a DT for CNC machines by monitoring product quality and system reliability in real-time, improving the capitalized knowledge of the whole system, and increasing the resiliency of manufacturing equipment. Hehenberger et al. [42] presented a case study where CPS and IoT have been integrated in wind turbines for condition monitoring reasons. Here, a Genetic Algorithm (GA) was exploited to reduce the number of variables to be managed by the CPS. Finally, Schroeder et al. [43] exploited CPSs and simulation (under the form of a DT) for monitoring the health level of a valving system. Subsequently, Schroeder et al. [44] upgraded the original model by exploiting AR and web services for supervising an oil tank. 


\subsubsection{Featured Application Cases: I4.0 Technologies Supporting WEEE Management Practices}

WEEE and CE are emerging topics attracting great interest in the fields of environmental science and engineering $[45,46]$. In order to strengthen the value of the current work, application cases focused on the same sector have been grouped in this sub-section. Here, the experts focus on remanufacturing and disassembly. Goodall et al. [47] developed a discrete-event simulation approach to predict material flow behavior within remanufacturing operations, by utilizing data coming from digital manufacturing systems for updating and automatically modifying the simulated model and reflect the real system state. The simulation approach has been tested in a WEEE remanufacturing facility and data have been gathered through radio-frequency identification (RFID) traceability systems. Alqahtani et al. [48] discussed an advanced remanufacturing system based on a discrete-event simulation model. Here, a smart refrigerator-with embedding sensors and network connectivity for gathering and exchanging data-was monitored in order to plan its refurbishing and repair. Subsequently, Joshi et al. [49] upgraded the original remanufacturing system by also considering the disassembly stage. RFIDs have been exploited for recovering data from obsolete laptops and, basing on that, for deciding the best EoL strategy to adopt through a multi-criteria decision-making model based on linear physical programming. Bressanelli et al. [24] explored how CPS, IoT, and BDA can improve remanufacturing performances in terms of monitoring, data gathering and process optimization of washing machines. Marconi et al. [50] presented a method for calculating the disassembly times of target components in washing and coffee machines. Data mining has been used to derive corrective factors that are useful in designing new products. Sharpe et al. [51] described the adoption of CPSs in WEEE management processes. Here, RFID tags were exploited for gathering data from the cores and subsequently sent to graphical user interfaces (GUIs) to monitor and control their refurbishment. Finally, Wang et al. [37] discussed a novel DT-based system for WEEE recovery that supported manufacturing/remanufacturing operations. DT was exploited to develop a reliable cloud-based avatar of WEEE, thus constituting a CPS.

In trying to summarize the most important topics from the literature review presented above, it is possible to say that there are clear perspectives about how I4.0 technologies can be integrated in current $\mathrm{CE}$ practices by contributing to strategic decision-making processes, data collection, and sharing $[22,23,28]$. However, there is a clear lack of real application cases (e.g., adopting simulation tools, CPSs, IoT, and robots together) supporting WEEE management practices. Attention has been voluntarily focused on WEEE and these four I4.0 technologies, given the potential benefits coming from their integration in achieving better performances during disassembly, remanufacturing, and recycling processes. The following sections will present a real application case going in this direction, where simulation (under the form of VR and DT models) and a robot interact to optimize a WEEE disassembly process. Before presenting the model conceptualization and development, the results of the literature review related to the interaction between advanced simulation tools and $\mathrm{CE}$ practices is reported.

\subsection{Model Conceptualization}

Among the main effects of simulation on CE practices, the literature underlines (i) support on products remanufacturing - for example, in the form of decision-support tools-and (ii) improvement of efficiency in exploiting natural resources through the calculation of eco-efficiency indexes $[5,37,52,53]$.

According to Smith et al. [54], it is possible to identify two main categories of simulation applications in the manufacturing sector-(i) manufacturing system design simulation and (ii) manufacturing system operation simulation. These two categories are based on the same interpretation of the concept of simulation, only differing in the way they are used within a manufacturing environment. From one side, manufacturing systems design (furtherly subdivided into general system/facility design/layout and material handling design) mainly refers to long-term decisions with large impacts on costs and efficiency of manufacturing operations. From the other side, manufacturing system operation applications (furtherly subdivided in operations planning/scheduling and real-time control) refers 
to short-term decisions. Following this categorization, the paper presents the development of two simulation models for a disassembly process optimization (see Figure 2, below):

- A simulation model belonging to the manufacturing system design category (i.e., a virtual reality (VR)-based disassembly process configuration model);

- A simulation model belonging to the manufacturing system operation category (i.e., a digital twin (DT)-based real-time process optimization tool)

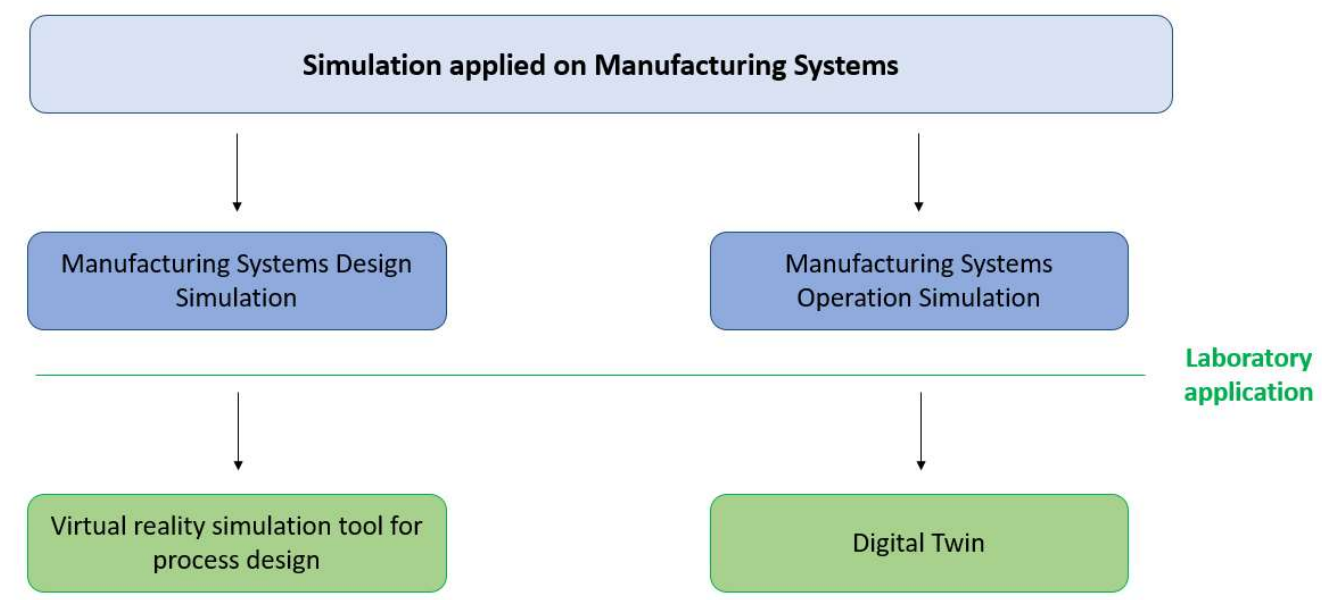

Figure 2. Simulation models schematization.

The first model focuses on facilities design, an important factor influencing the general performance of a manufacturing system. Facility layout design deals with the allocation of machines in a plant and can have a large impact on the effectiveness and efficiency of disassembly processes, increasing the ability to recover resources, and enabling their usage in new product value chains. An effective layout can reduce manufacturing costs and improve the system's performance. Discrete event simulation is an appropriate tool for evaluating the current layout, thus showing potential areas of improvement by evaluating different layout alternatives $[54,55]$. The second model focuses on the real-time control of a manufacturing system. This way, simulation is used as a tool for monitoring what happens in the system during the disassembly process, trying to optimize energy consumption and valuable materials recovery. Here, the simulation is exploited for easing real-time decision making. However, the use of simulation as a basis for a real-time system control is still a hard task due to response time, data collection, and aggregation issues, making it an emerging field of research within manufacturing systems [55-57].

\subsection{Models Development}

\subsubsection{The Industry 4.0 Lab of Politecnico di Milano}

The reference application case described in this paper was implemented at the Industry 4.0 Lab of Politecnico di Milano, pertaining to the research activities funded by the H2020 FENIX project. The Industry $4.0 \mathrm{Lab}$ is one of the few pilot plants in Italy fully focused on demonstrating the benefits coming from the introduction of I4.0 technologies in manufacturing. The core of the lab is constituted by a fully automated assembly (manufacturing) line developed by Festo ${ }^{\circledR}$ Didactics(Esslingen, Germany) [58], which is able to assemble a simplified version of a smartphone. This product is made up of several components:

- Front cover;

- Back cover;

- Fuses; 
- Printed circuit board (PCB).

The assembly line is made up of seven modular workstations, each of them controlled by a programmable logic controller (PLC). Here, services can be instantiated on each component and phase, both in terms of operations (by interacting with the MES) and energy consumptions (by interacting with the energy server). Different CPS and IoT infrastructures (infrared sensors, inductive sensors, RFIDs, quick response (QR) codes and barcode systems, and VR/AR system) allow users to track and trace the production flow in terms of single component or pallet.

Within the H2020 FENIX project, the Industry 4.0 Lab has been exploited for demonstrating how CPSs, IoT, AR/VR, DT, and robots can be used together for testing, managing and optimizing a WEEE disassembly process. In order to ease its description, the application case has been split into two configuration tools:

- a VR-based configuration tool supporting the disassembly process reconfiguration and implementation; and

- a DT-based configuration tool for a real-time disassembly process monitoring and control.

\subsubsection{The VR-Based Configuration Tool}

Trying to fill in the existing gap regarding application cases matching together 44.0 and CE, a VR-based configuration tool was developed for supporting disassembly processes reconfigurability and implementation. The process reconfigurability allows the user to improve the ability to manage a higher product variety for disassembly, thus achieving better materials recovery. The baseline idea was to exploit a virtual environment in which to simulate and optimize a disassembly process before efficiently replicating it on the real world. To this aim, CIROS ${ }^{\circledR}$ Studio 6.0 was selected as the reference software [59]. The virtual disassembly process configuration tool was implemented following five steps:

- Disassembly line modelling;

- Disassembly process design;

- Robotic disassembly program coding;

- Disassembly workplan creation within the MES software and process simulation;

- Disassembly configuration uploading on the real system.

Disassembly Line Modelling

CIROS $^{\circledR}$ Studio 6.0 embeds all the libraries for simulating each workstation constituting the real line (including conveyors), plus those related to other workstations that could be added in the future. This way, when all the workstations have been modelled, it is possible to combine them and virtually replicate and test the real line. In addition, smartphone components and robot tools have been added in the model. Figure 3 shows both virtual and real lines at the Industry 4.0 Lab. 


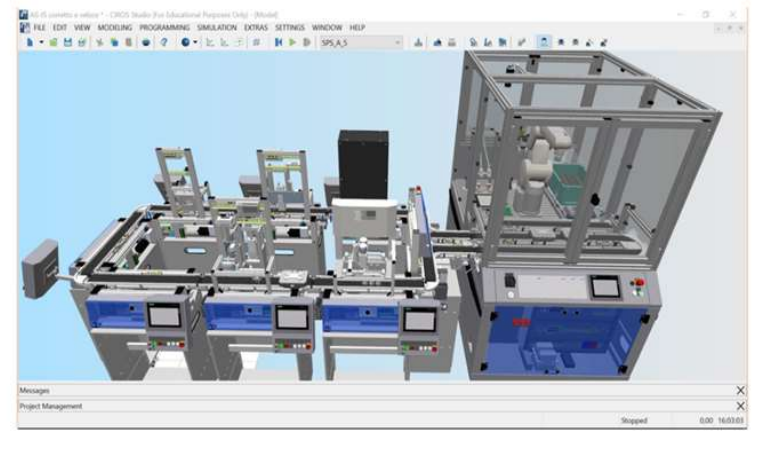

(a)

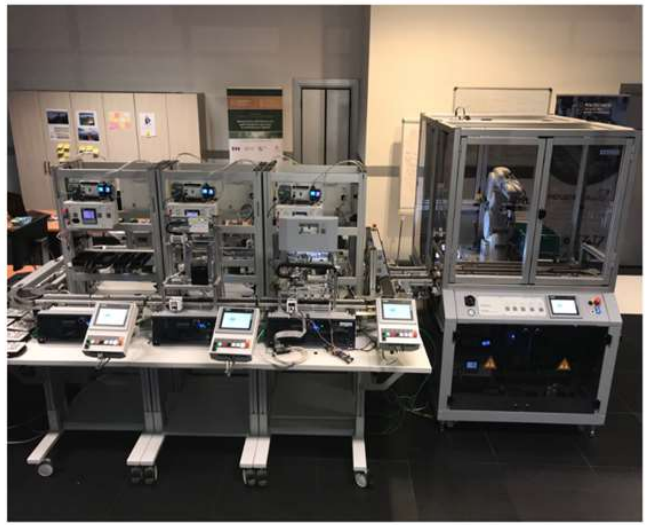

(b)

Figure 3. Virtual (a) and real (b) configuration of the line.

Disassembly Process Design

Starting from the final product (constituted by front cover, PCB, fuses, and back cover) - and considering constraints related with each workstation of the line-the disassembly process has been designed as follows:

- $\quad$ Step 1-Manual operation: this step refers to the manual removal of back cover and fuses by an operator, by interacting with the line through a human-machine interface (HMI). Given the initial structure of the line (originally designed for assemble products), it was not possible to remove the pressed-back cover. Specifically, robot tools do not currently allow for the removal of the pressed-back cover because it is needed to fix the product on the pallet.

- Step 2-Unscrewing: This step is performed by the drilling station, through the unscrewing of the front cover from the PCB.

- $\quad$ Step 3-PCB removal: This step is performed by the robot station by disassembling the PCB from the front cover and positioning it in a dedicated box. This step enables valuable components recovery (i.e., $\mathrm{PCB}$ ), making it available for further remanufacturing and recycling activities. This represents the core of the disassembly process, since the program performing this operation has been directly developed and tested in the simulation environment.

- Step 4-Final inspection: The last step is performed by the camera inspection station, which is responsible for checking if the $\mathrm{PCB}$ has been correctly removed from the front cover.

\section{Robotic Disassembly Program Coding}

The robot station is the most flexible one. According to specific types of production needed, it is possible to easily reconfigure it in CIROS ${ }^{\circledR}$. Considering the disassembly process, a dedicated code has been implemented in order to control the robot working cycle through a series of moving instructions. To this aim, Melfa-Basic V [59] has been selected as the reference robot programming language. Once the code was tested and validated in the virtual environment, it was uploaded to the real robot. In order to program the robot, it was important to understand which positions, tools, and movements are needed to perform the correct removal of PCBs.

Figure 4 shows the most relevant positions that can be assumed by the robot and the tools to which the robot arm can be jointed. 


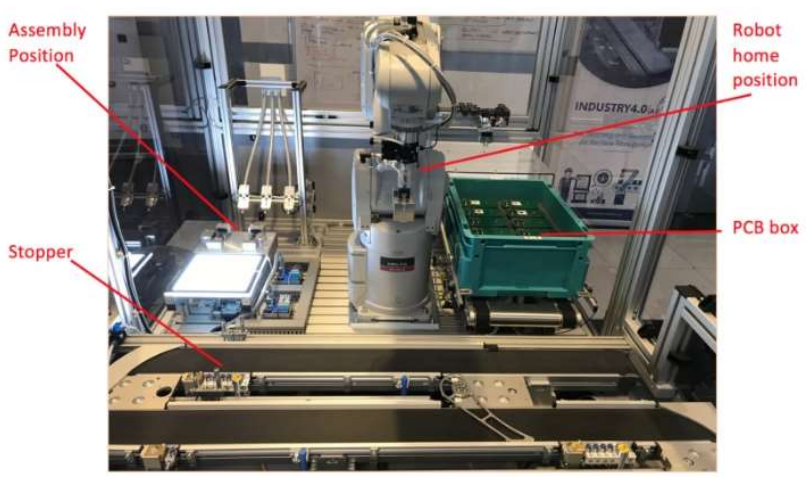

(a)

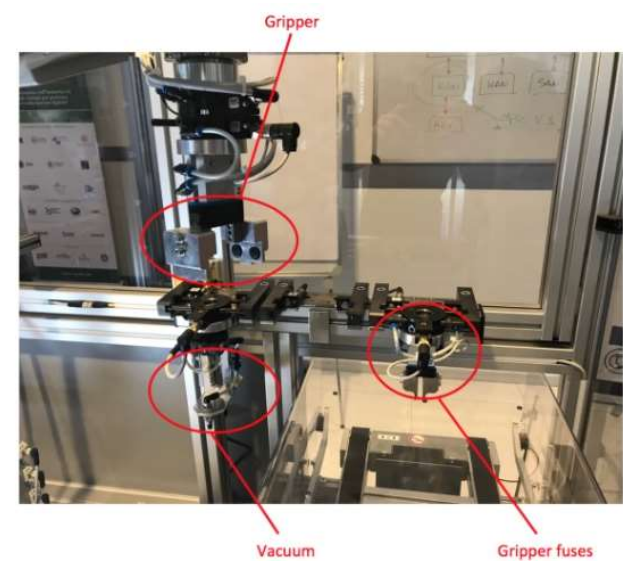

(b)

Figure 4. Positions (a) and tools (b) within the robot cell.

Disassembly Workplan Creation within the MES Software and Process Simulation

Coherently with the automation pyramid presented in Section 1, within the Industry $4.0 \mathrm{Lab}$, a MES is responsible for creating, managing, controlling, and launching the workplans. The Industry 4.0 Lab MES has many functions that enable the planning field of the automation pyramid. Substantially, this MES is specially prepared with a peculiar design for I4.0 learning platforms. It features an open database, and it can be written by external programs and read via Structured Query Language (SQL) commands by external programs. Furthermore, the individual controllers can communicate with the MES via Transmission Control Protocol/Internet Protocol (TCP/IP) communication protocol. The MES can be exploited for several purposes:

(i) Define and edit work order flows and process plans;

(ii) Read orders and update status;

(iii) Write allocation of the goods carriers to the order;

(iv) Create warehouse data and material buffer;

(v) Overall equipment effectiveness (OEE) calculation;

(vi) PLC and malfunction report generation, including graphic representation.

According to these points, before starting any kind of production, it is necessary to plan and develop a new workplan using the MES software. Within the virtual environment, the MES is responsible for launching orders. This is the reason why CIROS ${ }^{\circledR}$ is provided with its own MES, which communicates with the virtual line. In order to start the simulation, the creation of a disassembly work plan and its uploading to the MES is required. The disassembly workplan created within the MES reflects the four steps described for the disassembly process design.

Once the line is virtualized and the disassembly program of the robot is coded, the disassembly process can be simulated in $\operatorname{CIROS}^{\circledR}$ by running the dedicated workplan uploaded within the MES. This way, it is possible to test and control the sequential operations constituting the workplan, both in terms of errors present in the workplan (if so, the simulation stops) and moving instructions sent to the robot (automatically optimized by the software).

Disassembly Configuration Uploading on the Real System

Once the code and process have been virtually optimized, $\operatorname{CIROS}^{\circledR}$ allows a direct connection between virtual and real contexts, by setting out the communication port. This way, the code can be transferred from virtual to real environments. At the same time, the workplan uploaded within the MES starts the production on the real system.

The adoption of a VR-based simulation tool together with advanced manufacturing systems allows us to comprehend how it is possible to support the transition toward CE practices with I4.0 
technologies, reconfiguring a fully automatized manufacturing line originally designed for assembly processes. After having focused the attention on design and optimization, the following sub-section will describe in detail how to develop a DT simulation tool.

\subsubsection{The DT-Based Real-Time Process Optimization Tool}

The real value of the I4.0 paradigm is the new way through which information is managed across the different automation pyramid levels. To this aim, CPS can lead to this transformation without changing the whole perspective [15]. A CPS-based structure of an automated system can follow the 5C (connection, conversion, cyber, cognition, and configuration) architecture available in the literature [19], constituted by two functional elements:

- Advanced connectivity, ensuring real-time data acquisition from the physical world and information feedback from the cyber space;

- Intelligent data management, analytics, and computational capability, constructing the cyber space.

According to reference [19], the 5C levels shown in Figure 5.enable the implementation of a CPS starting from the data acquisition stage.

- Connection-data are acquired from machines and their components;

- Conversion-acquired data are transformed into useful information;

- Cyber-information is exploited for building a virtual copy of the real system;

- Cognition - the acquired knowledge is shown to expert users and compared with available information for a self-comparing capability, which improves the decision-making process;

- Configuration - the feedback layer acts on the real system as supervisory control.

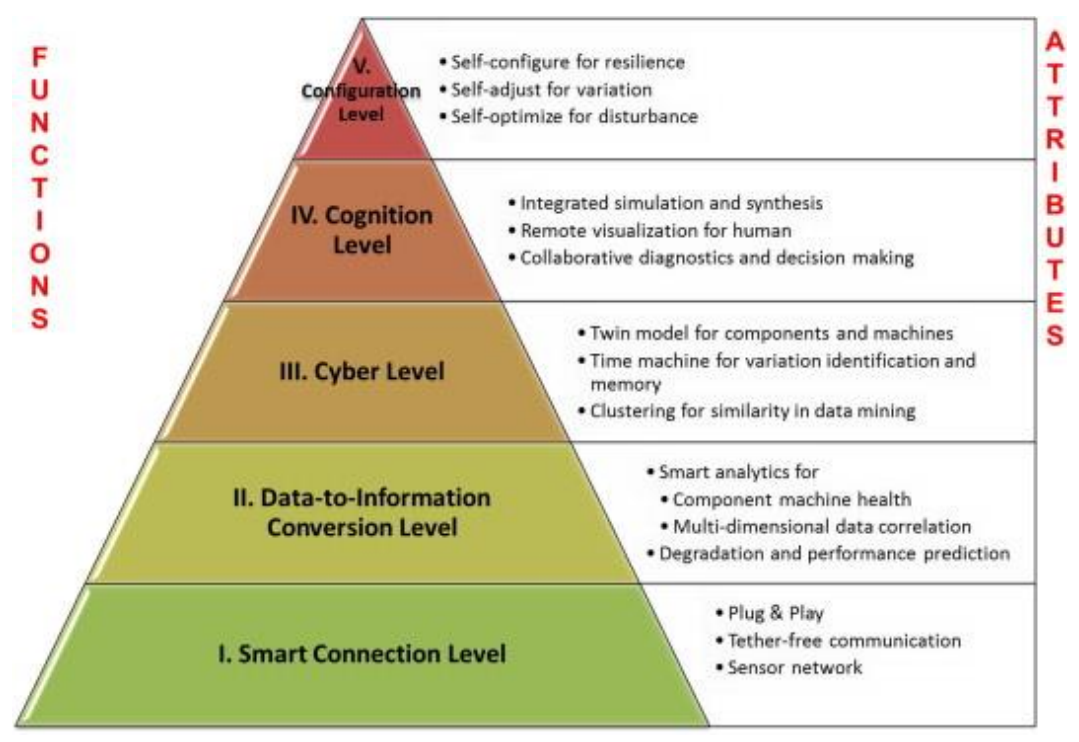

Figure 5. Cyber Physical System (CPS) 5C Architecture [19].

CPS being at base of the pyramid (field level) leads to a convergence of physical and digital worlds within the Digital Twin [15] by allowing a real-time simulation of processes, material flows, and energy consumptions [60]. The Industry 4.0 Lab DT has been developed by exploiting the OLE for Process Control (OPC) Unified Architecture (OPC-UA) communication protocol. This is a Machine-to-Machine (M2M) communication protocol compliant with the IEC 62541 standard allowing a real-time information exchange with sensors and actuators of the real system between machines and an external simulation environment [61]. OPC is a technology commonly used in PLC, Distributed Control System (DCS), and Supervisory Control and Data Acquisition (SCADA) devices as a basic 
communication platform for integrating, supervising and controlling data. OPC-UA uses client-server architecture with clearly assigned roles:

- Servers are applications sharing information basing on the OPC-UA information model. Each server defines an address space containing nodes of the OPC-UA model. These nodes represent physical or software objects.

- Clients are applications retrieving information from servers, by browsing and querying the information model.

This way, the OPC-UA protocol enables communication between the industrial equipment and systems for a continuous data collection and control by creating a virtual mirror of the system. This mirror is the cyber part of the physical plant through which machine data can flow, and the process simulation can be carried out. Moreover, the information flow coming (going) from (to) the field (e.g., sensors states and variables values) can be read and managed in real-time also through generic numerical computing and engineering tools, such as MATLAB ${ }^{\circledR}$ and Simulink ${ }^{\circledR}$. Since all the modules in the Industry 4.0 Lab are equipped with PLCs representing OPC-UA nodes, it is possible to

- Create a real-time connection with workstations-by exploiting the OPC-UA Toolbox in MATLAB ${ }^{\circledR}$ — by accessing to live and historical data directly from MATLAB ${ }^{\circledR}$ and Simulink ${ }^{\circledR}$;

- Read, write and log in OPC-UA directly from PLCs.

- In general terms, through the Industry 4.0 Lab DT it is possible to

- Identify possible machine states (e.g., errors, failures and downtimes). It is possible to consider CPS as a way to enable either better lifecycle management of products or the development of new services, especially for maintenance reasons [62];

- Identify sensors and actuators states/values (e.g., presence of products, temperature or air consumption), allowing a better monitoring of resource usage within disassembly process (e.g., energy consumption, scrap rates and waste management);

- Monitor and control operations performances;

- Real-time analysis of signals;

- Define maintenance plans of machines;

- Store all the gathered data;

- Execute data analytics on operational and energy parameters.

As an example, in Table 1, the path identification of a specific node (sensor xBG1) of the Magazine Front Station (IP address: 10.48.134.20) through UA Expert is reported. This sensor is used to know if the carrier is in a working position within the station.

Table 1. Command steps to find and connect an OLE for Process Control Unified Architecture (OPC-UA) variable in MATLAB ${ }^{\circledR}$.

\begin{tabular}{cc}
\hline Command Step & Practical Step \\
\hline $\begin{array}{c}\text { serverList }=\text { opcuaserverinfo ('10.48.134.20:4840') } \\
\text { uaClient }=\text { opcua(serverList) } \\
\text { connect(uaClient) }\end{array}$ & $\begin{array}{c}\text { Connect to the server with the right IP address data } \\
\mathrm{f}=\text { getNamespace(uaClient) } \\
\text { Creation of the client }\end{array}$ \\
$\begin{array}{c}\text { Connect the created client to the server } \\
\mathrm{k}=\text { findNodeByName(f,'plcMagazineFront','once'); } \\
\mathrm{I}=\text { findNodeByName(b, 'Inputs','-once'); }\end{array}$ & $\begin{array}{c}\text { Using the address founded in UA Expert, the user } \\
\text { knows the sequence of node to reach the right }\end{array}$ \\
\end{tabular}

Once the connection for the variable real-time reading is done, the next step is to try to extract these values using the MATLAB ${ }^{\circledR}$ function in Simulink ${ }^{\circledR}$. To do this, Level-2 MATLAB ${ }^{\circledR}$ S-functions are used. This kind of function allows us to create custom blocks with multiple input and output ports capable of handling any type of signal produced by a Simulink ${ }^{\circledR}$ model. The MATLAB ${ }^{\circledR}$ function 
comprises a set of call-back methods that the Simulink ${ }^{\circledR}$ model invokes when updating or simulating the model. The implementation of this call-back method, in turn, determines the blocks attributes (e.g., ports, parameters and states) and behavior (e.g., the block outputs as function of time and the block inputs, states and parameters). In this specific case three types of call-back methods are used, which are the ones that allow the extraction of the values of the sensors and actuators present on the line and the creation of the blocks related to this sensor and actuators in the Simulink ${ }^{\circledR}$ environment. These blocks compose the DT model of the line. The three main parts used in the Level-2 MATLAB ${ }^{\circledR}$ S-functions are

(i) Setup-in this part, the number of inputs and outputs of the function, their datatype, complexity, and dimensions are defined. Since the model crated is a Discrete Event Simulation, this method includes also the specification of the sample time required for the lecture of sensors values.

(ii) Start-this part performs the initialization activities that the S-function requires, such as allocating memory and setting up user data. This method, as reported in Table 1, allows the connection to the server of interest only at the start of the simulation in Simulink ${ }^{\circledR}$. Then, after having properly defined the address of each node that it is required to extract from the server, this step allows Simulink ${ }^{\circledR}$ to relate to the specific nodes in real-time.

(iii) Outputs-this part calculates the S-function's outputs at the current time step and store the results in the run-time object's OutputPort(n).Data property.

The Level-2 MATLAB ${ }^{\circledR}$ S-functions that has been used to read the Carrier ID is reported in Figure 6. Using this function in Simulink ${ }^{\circledR}$, it is possible to monitor in real-time the state of these sensors and actuators in this virtual environment. Hence, in order to create the DT of the production line, it is possible to combine the sensors and actuators values using simple MATLAB ${ }^{\circledR}$ functions to reproduce, for example, the machine states (idle, working, energy saving, etc.) or to evaluate the energy consumption of each station.

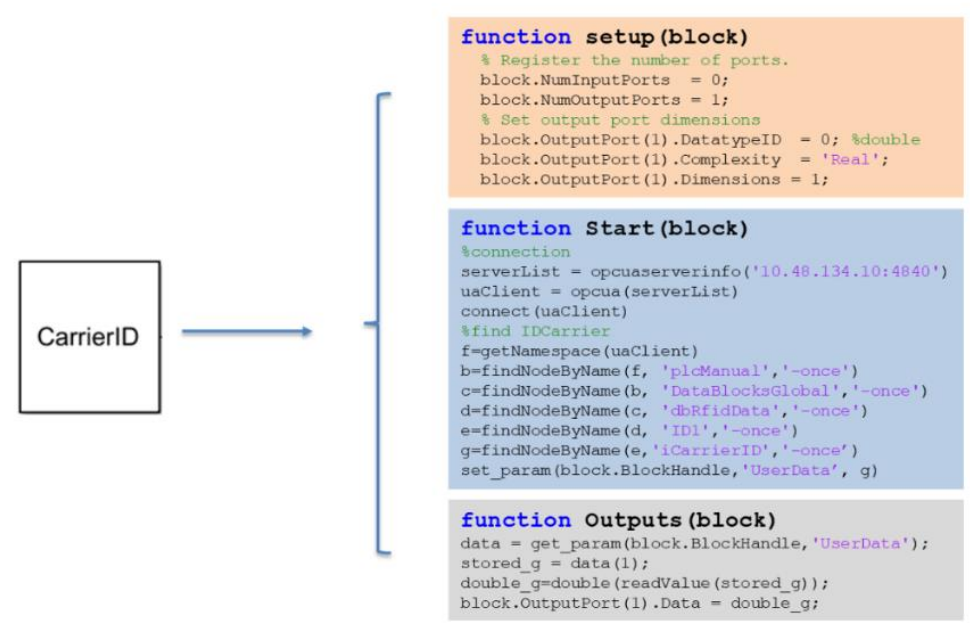

Figure 6. Level 2 MATLAB ${ }^{\circledR}$ S-Function code to read the Carrier ID value from the server.

The sequence of activities needed to do that is the same for all stations and can be summarized as follows:

1. Identification of the possible machine states;

2. Identification of sensors and actuators useful in order to reproduce the machine states identified in the previous step and their combination for the model;

3. Analysis of the signal in real-time;

4. Storage of the data.

Table 2 summarized the five different states that have been identified with the correspondent identification number. 
Table 2. Machine stat.

\begin{tabular}{crc}
\hline Machine State & Description & Output \\
\hline Idle & $\begin{array}{c}\text { The conveyor is moving but no operation is performed. } \\
\text { The machine is waiting for a piece to be processed. }\end{array}$ & 1 \\
\hline Working & The machine is performing an operation. & 3 \\
Error & $\begin{array}{l}\text { For each of the station of the line, a specific kind of fault have } \\
\text { been identified. The machine is blocked due to abnormal } \\
\text { behavior and shows an error message in the Human-Machine } \\
\text { Interface (HMI). Only when the fault is fixed and the operator } \\
\text { respond to the error message on the HMI, the working resumes. }\end{array}$ & 4 \\
Emergency button & $\begin{array}{l}\text { State of the machine that can be classified as a fault state, } \\
\text { in which the normal behavior of the machine is stopped due to } \\
\text { the fact that the operator has triggered the emergency button. }\end{array}$ & 3 \\
\hline Energy-saving mode & $\begin{array}{l}\text { The machine is on, but the belt is not moving to save energy } \\
\text { when there is no piece to work immediately. }\end{array}$ & 5 \\
\hline
\end{tabular}

The sensors belonging to the belt system of the stations used for the identification of the machines states are described in table below (see Table 3).

Table 3. Belt system sensors.

\begin{tabular}{|c|c|c|}
\hline Sensor & Description & Values \\
\hline xQA_A1 & Sensor used to know if the belt is moving or not & 1 if the belt is moving \\
\hline xBG1 & $\begin{array}{l}\text { Sensor used to know if the carrier is in the working position of } \\
\text { the station }\end{array}$ & $\begin{array}{l}1 \text { when the carrier is ready to } \\
\text { be processed }\end{array}$ \\
\hline xBG5 & Entrance sensor of the machine's belt & 1 when carrier moves over it \\
\hline xBG6 & Exit sensor of the machine's belt & 1 when carrier moves over it \\
\hline xMB1 & Stopper sensor, that releases the carrier from working position & 1 when activated \\
\hline
\end{tabular}

Then, MATLAB ${ }^{\circledR}$ functions have been implemented in order to combine these sensors and reproduce a specific machine state. The example of the emergency button state with the combination of its related sensors, is described in Table 4 . The emergency button can be used by the operator to immediately stop the station when it is needed (see Table 5).

Table 4. Emergency button sensors.

\begin{tabular}{cc}
\hline Sensor & Description \\
\hline xPF1 & It is set to 1 until the operator checks the error on the HMI \\
xPF3 & When is set to 1, the green light of the 'Start' is turned on to show that the operator must \\
press it to resume the work & Set to 1 when the emergency button is released \\
\hline
\end{tabular}

Table 5. Actions definitions of emergency button triggering.

\begin{tabular}{ccccc}
\hline xSF5 & xPF1 & xPF3 & Action & Em.Button \\
\hline 0 & 0 & $0 / 1$ & Button triggered & 1 \\
1 & 0 & $0 / 1$ & Button released & 1 \\
1 & $0 / 1$ & 1 & Wait for start & 0 \\
1 & 1 & 0 & Start triggered & 0 \\
\hline
\end{tabular}

The sensors and actuators identified are common to all the stations. This implies that the schematization of the idle, working, and emergency Button states is the same for all the machines. What differs from one station to another is the definition of the error state, since each of the stations 
of the line performs a specific operation on the product. This means that different failure modes are linked.

Once all these steps have been done for each of the seven stations of the line, the combination of all the models allows us to create the final DT of the line exploiting the Simulink ${ }^{\circledR}$ SimEvent ${ }^{\circledR}$ blocks. In this way, it is possible to have a graphical representation of the assembly-disassembly line. It is also possible to see, for each station the number of worked pieces, if there is any piece working and the number of pieces attending to be worked, as reported. Moreover, the DT represents a useful tool that allows for the exploitation of a database for post-processing of data to compute KPIs needed to make decisions.

\section{Results}

\subsection{Energy Consumption Optimization}

\subsubsection{Energy Data Acquisition}

The model was developed in a practice environment with the aim of obtaining the final consolidated Waste from Electrical and Electronic Equipment (WEEE) disassembly process optimization. Among the waste sources produced by human activity, electronic waste (e-waste) is one of the most important, in terms of both volume and growth [45]. This was the first research field where the simulation models were tested regarding their energy efficiency (understood as process energy consumption optimization through digital solution). One of the problematic issues that the industrial sector faces as the largest consumer of electricity is $\mathrm{CO}_{2}$ production and its related environmental impacts. For this reason, the energy sustainability of disassembly processes helps to justify their implementation instead of different solutions within the product's EoL phase (e.g., incinerator or landfill). However, limited resources and high costs lead energy production not to grow at same ratio, resulting in a demand-supply mismatch. Considering this gap, energy suppliers and consumer are working to keep demand at a secure level. As an energy consumer, the industrial sector can use the available energy more efficiently.

Since each of the stations of the Industry 4.0 Lab is provided with two PLCs (one responsible to manage the process and one responsible for monitoring energy parameters), it is possible to connect the DT to the energy server to gather energy consumption data from each station. The energy server connections are possible through the Level-2 MATLAB ${ }^{\circledR}$ S-function. From this server three data are extracted - the instantaneous power consumed by the station (rActivePower), the pneumatic system pressure of the station (rPressure), and the air flow rate during the working condition (rFlow).

These variables have been identified to monitor and control the energy consumption and the working condition of the line. At this point, in order to match the machine states with the respective energy consumption, we created an accumulator function. This function allows us to associate the respective energy consumption to each one of the five machine states identified in the model. The function takes as input the machine state, the instantaneous power consumed by the energy box and the sample frequency of the simulation. It gives as outputs

- The time spent by the machine in each state $t_{i}[s] ;$

- The mean value of the power consumed in each state during the simulation $\mathrm{P}_{\mathrm{i}}$ [W];

- The energy consumption in each machine state (Idle, Working, Fault, and Energy Saving) during the simulation $E_{i}=P_{i} \times t_{i}$.

\subsubsection{Energy Data Analysis}

The simulation can improve efficiency when exploiting resources-for example, through the calculation of eco-efficiency indexes. In this way, the DT allows for the real-time interaction with the system in order to monitor and control the line. Basically, it uses data extracted directly from the shop-floor, enabling their collection. Then, data analysis can be applied to these data, giving benefit to the decision-making process. An energy management application exploiting the two simulation tools 
presented in this paper has been created by developing a proper energy consumption indicator whose value has been elaborate through the DT disassembly simulation runs. To achieve these objectives, an energy-based Overall Equipment Effectiveness (OEE) was developed and tested. The DT simulation and energy-based OEE need a proper graphical user interface to be more user friendly and support easier decision making. The energy Graphical User Interface (e-GUI) development allowed for the real-time monitoring of the stations, enabling the immediate identification of what happens in the production line. Finally, these energy tools have been applied to different production processes in order to evaluate their results and differences.

From the literature, it has emerged that many KPIs are continuously calculated in order to evaluate production system performance. In particular, reference [63] underlines that the OEE is a widely used performance indicator in manufacturing industries. As reported in their work, the increasing industrialization provides means to automatically acquire manufacturing data and analyze them. Therefore, companies are investing in MES where the OEE measurement is a central part, as happens also with the MES of Industry 4.0 Lab. The OEE is a KPI developed to investigate the performance of the production system considering the speed, quality, and breakdown of the systems. It is a time-based approach aiming to measure the direct time related to the production pieces with respect to the total production time.

Starting from the definition of OEE, an energy-based OEE (e-OEE) applied to the line, in order to evaluate its energetic performance, has been introduced. The definition of the e-OEE derives from the definition of the classic OEE, with an effort in translating the time-based indexes presented before into energy-based indexes. In order to introduce this concept, a distinction between the type of energy consumed in the line is necessary. The following two types of energy have been identified:

- Active energy - this is the energy from which, in theory, is possible to extract added value for the final product (both for assembly and disassembly processes); and

- Passive energy - this is the energy wasted by the line, i.e., it does not produce any added value to the final product.

Active energy includes types of energy consumption related to three machine states-Idle, Working, and Failure energy consumption. During the daily working cycle, the overall energy consumption of the stations in the Industry 4.0 Lab is equal to the sum of different contributes, both active and passive, as it is possible to see from Figure 7.

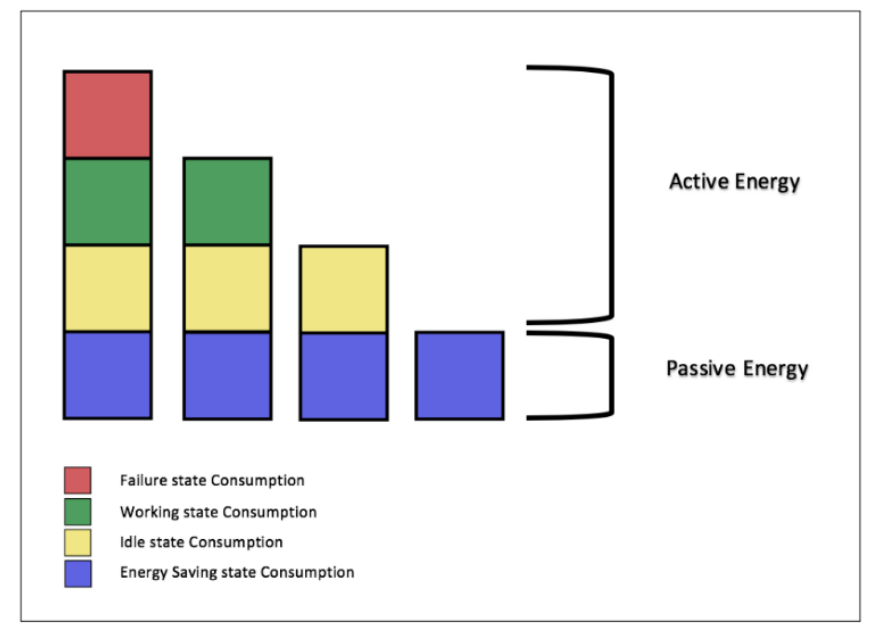

Figure 7. Graphical representation of energies type.

The traditional time-based OEE is composed of three terms-availability, performance, and quality. At the basis of this KPI there are three important time concepts-planned production time, run time, and working time. From an energetic perspective, these concepts are substituted with 
- Total energy consumption - this is equal to the overall energy consumed by the line (or a machine if applied to a single station). It is the sum of all the four components shown in Figure 7.

- Operative energy consumption-this is equal to the Total energy consumption minus the passive energy. in other words, it is the sum of the active energy;

- Working energy consumption - this is the sum of the energy consumption in the working state (or the working consumption of the single machine).

It is also possible to define the different coefficients that are used to calculate the e-OEE:

- e-Availability-under the energetic perspective, the availability evaluates how much of the total energy consumption is used to perform active operation on the processed piece. The availability is equal to

$$
\text { e-Availability = (Operative Energy Consumption)/(Total Energy Consumption) }
$$

- e-Performance-from an energetic perspective, the performance evaluates how much of the active power is effectively used to perform operations on the processed product. The performance is equal to

$$
\text { e-Performance }=(\text { Working Energy Consumption }) /(\text { Operative Energy Consumption })
$$

- Quality-defined in the same way as for the time-based perspective so as the ratio between accepted product over the number of products produced:

$$
\text { Quality }=(\text { Accepted products)/(N. of produced/disassembled products) }
$$

The e-OEE indicator is calculated as

$$
\text { e-OEE }=\text { e-Availability } \times \text { e-Performance } \times \text { Quality }
$$

e-OEE is defined as the ratio between the energy consumed for producing goods of approved quality and the overall energy consumption of the line. As previously discussed, the e-OEE can be applied both to the whole line or to a single station according to the analysis. In fact, if applied to the whole line, this KPI can monitor overall energy consumption, giving an overview on how much of the energy consumed in each time period is effectively used for the production and not wasted. Also, the calculation of the e-OEE for different processes gives us the opportunity to understand which are the most performing under an energy-based approach, giving the possibility to understand which are the critical ones that require some kind of improvement for their optimization.

On the other hand, the e-OEE is thought also to be applied to each station in order to make a comparison between them. In fact, fixing the monitoring time, it results in the e-OEE being normalized to each station since all the coefficients presented in the formula are only function of the station in which it is applied. In this way, it is possible to evaluate which is the critical station from an energetic point of view. Once it has been identified, some improvements could be identified in order to optimize its energetic behavior.

\subsubsection{Energy Data Interface}

In order to link together what has been discussed regarding the energy monitoring and management application, a Graphical User Interface has been developed using the MATLAB ${ }^{\circledR}$ GUI. The GUI is connected to the DT of the line, and it can give the user an overview of the most relevant data for the process control. This links together the main scope of the two simulation tools introduced because, once the disassembly process has been updated on the real line, the GUI is able to show and extract from the DT the data related to this type of configuration in a user-friendly way. It also calculates the e-OEE for both assembly and disassembly processes, giving the possibility to evaluate which is the most performing from an energetic point of view.

The developed GUI contains 
- Start and Stop buttons-by clicking these two buttons, it is possible to start and to stop the simulation with the DT while data are extracted;

- Analyze button - when the simulation is stopped, it is possible to calculate the e-OEE;

- Energy button-with this button, it is possible to gain access to a graphical interface that plots the power absorbed by each station as function of the time;

- Machine values-for each machine, it shows the state, the CarrierID on which the piece is processed, the number of the process (given from the MES), the actual power consumption, and all the energy consumption discussed before. Also. the result of the camera inspection is available;

- Database generator-this button is available for each station and allows for the generation of the excel file with data gathered.

Figure 8 shows how the GUI appears. It is a user-friendly interface where each section is referred to a machine of the line. The main advantages of the GUI are

1. The data are clearer, more readable, and do not require the user to be able to read the code that lead to the creation of the DT;

2. It is possible to keep the stations under control in real-time and identify immediately what is happening in the production line;

3. It is possible to have a direct evaluation of the energetic performance of the line.

Together with the GUI, an interface that monitors the energy consumption of the line in real-time has been also developed. It is possible to plot this energy monitoring interface using the energy button of the GUI. Basically, it gives the same information of the previous interface, but it allows the user to have a graphical plot of the energy consumption as function of the time, if required. This interface is a tool linked to the DT since it is related to the data extracted from it. It is linked to the actual energy consumption of each station.

\subsection{Test of the Disassembly Process}

The process monitoring tool described above has been tested through different production plans within the Industry 4.0 Lab. The validation activity was carried out through different test, regarding both assembly and disassembly processes. In order to evaluate all the possible scenarios, a simulation with an error state for some stations has also been included. Here, the disassembly process is considered, exploiting in this way both the simulation tools presented in this paper. Starting from a generic production plans composed of three products already assembled, the disassembly process was performed to test the system reconfigurability. The main difference between the assembly and disassembly processes implemented on the line is that, in the second scenario, not all the stations are exploited. For this reason, it is expected that the e-OEE will be lower if compared to the one of the first configurations, since three station are not used in the production cycle of the processed pieces. Then, the e-OEE of this station will be equal to 0 . Figure 8 shows the results obtained at the end of the simulation through the GUI.

As expected, the e-OEE of the magazine front cover, magazine back cover, and press stations is equal to 0 since these stations do not perform any kind of operations, and the energy consumed by these stations is wasted. Overall, the disassembly process is more energy consuming compared to assembly, resulting in a lower value of e-OEE. This is justified by the fact that the line has been designed to perform the assembly process, and so the disassembly process it is not optimized from an energetic point of view. 


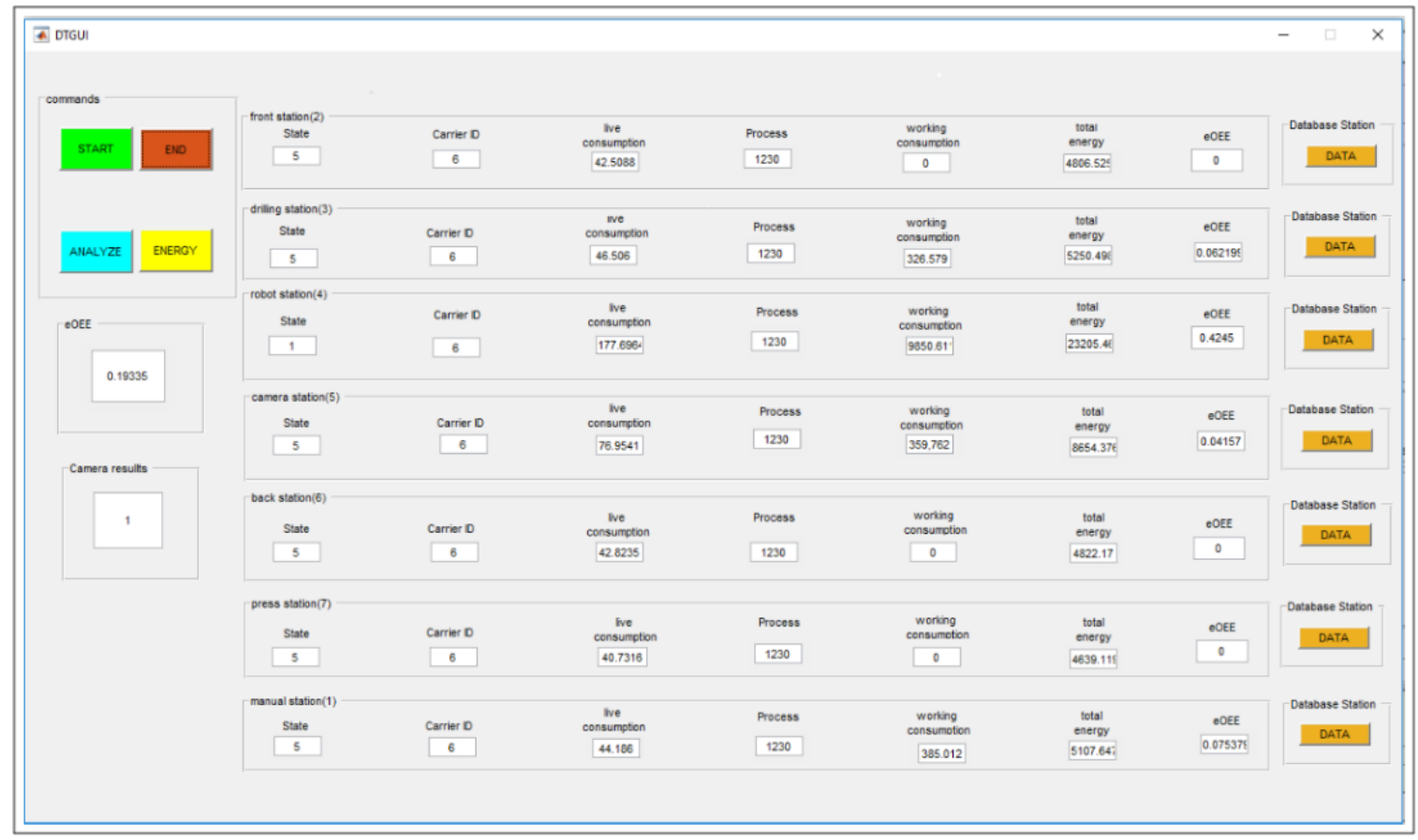

Figure 8. Disassembly process graphical user interface (GUI).

Since disassembly is an important part of remanufacturing systems for reuse and recycling purposes, automation and digitalization have a growing number of applications in the area of WEEE. For this reason, reconfigurability of disassembly systems represents an important paradigm of automated disassembly system that uses reconfigurable manufacturing technology for fast adaptation to changes in the quantity and mix of products to disassemble [64]. However, there are still many barriers in reverse production process adoption due to the complexity of the most of manufacturing systems and to the high number of product and components to be treated. As pointed out in reference [65], system reconfigurability can be classified in terms of the levels where the reconfigurable actions are taken-(i) at lower levels is mainly achieved by changing hardware resources and (ii) at the higher levels when is mainly achieved by changing software resources and/or by choosing alternatives methods or organization structures by flexible people. As demonstrated by the experiments, the changes at lower levels can imply a process performance decrease, representing a technical barrier to CE adoption at the factory level.

\subsection{MES Integration}

In the literature, few studies focus on either disassembly or reuse, apart from a few papers that are related to I4.0 technologies in general as good support for disassembly [5]. Among these, digital technologies are exploited for disassembly process optimization and disassembly sequence planning. Coherently with this, an application that aims to integrate the MES software with the DT in order to close the information flow between the simulation tool and the shop-floor has been created. This integration introduces bi-lateral communication, enabling the possibility to have control capability from the digital side to the physical side of an asset. In this way, not only is it possible to monitor real-time the process and gather the data in order extrapolate information, but they can also act autonomously on the process. This can enrich the simulation models with decision-making ability related to shop-floor level events in the production facility.

First, a way to send commands to the MES and get information by it was performed manually in MATLAB $^{\circledR}$. Second, the focus switched on developing the same functions on Simulink ${ }^{\circledR}$. Using this tool, a way to automatically communicate with the MES from the DT was conceived. The procedure of sending information to the MES via MATLAB ${ }^{\circledR}$ is one of the easiest and the most immediate to 
implement, since the only steps to follow are first, create a TCP/IP object to connect to the MES server; and second, send the relative string to request the performing of a specific task to the MES.

As in the MATLAB ${ }^{\circledR}$ library, the use of the TCP/IP-embedded function was needed [66]. The use of strings can be done for any communication using the standards on the library available.

The operation to get the information that the PLC sends back is more delicate and needs more steps. The basic flow to send information to the MES server, and so to the PLC, is exactly the same as the one reported in Table 6.

Table 6. Basic steps to send commands to the Programmable Logic Control (PLC) via Transmission Control Protocol/Internet Protocol TCP/IP communication protocol in MATLAB ${ }^{\circledR}$.

\begin{tabular}{|c|c|c|}
\hline Step & Code & Description \\
\hline 1 & $\mathrm{t}=$ tcpip (“RemoteHost", Remote Port); & $\begin{array}{l}\text { Creation of TCP/IP object, connected to a remote host } \\
\text { (IP address of the MES server) with a remote port }\end{array}$ \\
\hline 2 & fopen $(t)$ & Connect the TCP/IP object to the host \\
\hline 3 & fprintf $(t$, sprint $(“ \ldots$ ". ")); & $\begin{array}{l}\text { Write in the TCP/IP object }(\mathrm{t}) \text { the formulation of the } \\
\text { string by creating a string variable (sprintf) }\end{array}$ \\
\hline
\end{tabular}

The difference lies in the fact that the third step should contemplate a string capable to "get" information. A block-based sequence of the process to get information is given in Figure 9. If the "send" type just transmits control data to the PLC, the "get" kind can interrogate the PLC about some specific information, storing it in a certain object for it to be retrieved by the user in a second moment. So, by applying the procedure in Table 5 with the proper string, the TCP/IP object will be created and stored on the MATLAB ${ }^{\circledR}$ workspace.

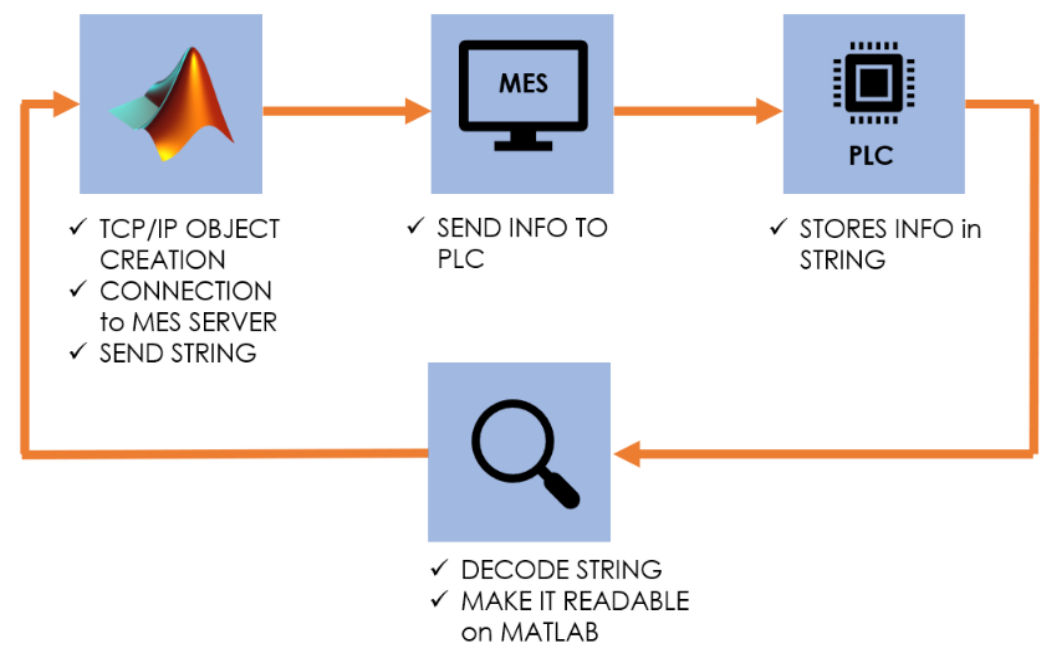

Figure 9. Operations performed by the "get" information model.

Moreover, the creation of a connection through which the DT can communicate with its physical counterpart in both directions is proposed with the definition of an optimization framework. The optimization aims for disassembly processes that can be reached using the VR-based simulation model, and the DT that is tightly integrated to its physical counterpart. This re-scheduling and disassembly framework proposes an integration of physical and digital sides with the objective to optimize the production process in terms of reactive disassembly in cases in which compliance with respect to certain production standards is not respected. Even in this case, the modelling of a tailored simulation-based DT is proposed to deal with the reactive scheduling of disassembly processes. The purpose of this application framework is to practically demonstrate the integration of I4.0 technologies for the disassembly process optimization and digitalization in order to improve the rates of waste generation and product components restoration. 
In this case, the framework in Figure 10 has a simpler way of working:

- The order, already assembled, arrives at the station in charge of checking compliance. If conformity is verified, the process continues, otherwise it does not.

- When compliance is not verified, the DT aborts the current work plan and acts by reactively scheduling a disassembly plan on the same piece.

- For this framework, optimization lies in the autonomous reactive scheduling of a disassembly plan, whether certain compliance conditions are not met.

- An overall scrap reduction with the possibility of materials recovery.

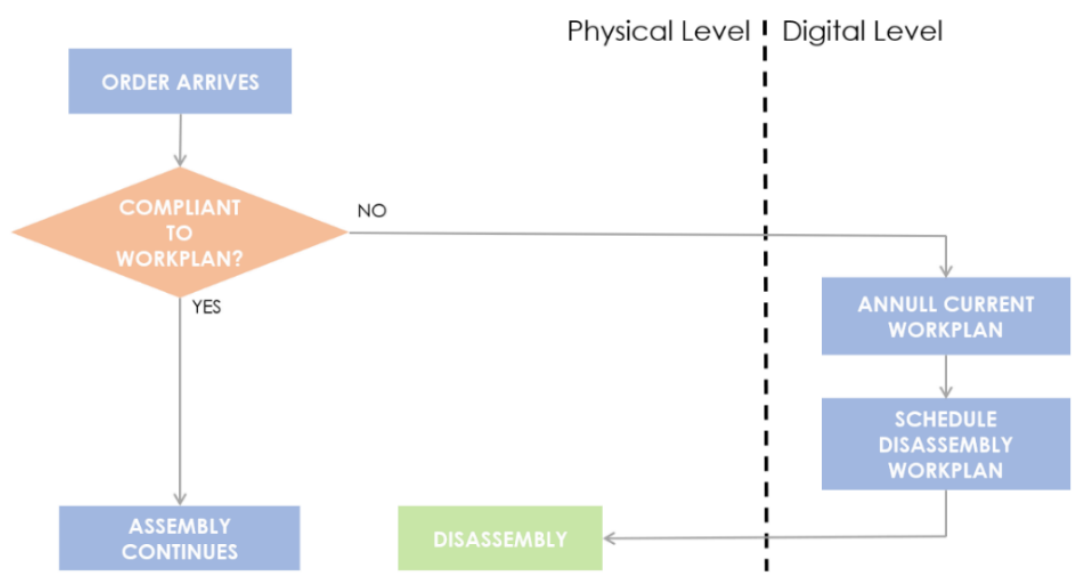

Figure 10. Framework of integrated management of disassembly with the simulation tool.

The function to be embedded in the DT is one that processes the values coming from the other function of the DT and identifies the conditions in which the reactive scheduling of a disassembly process is necessary. In fact, in order to avoid the scrapping of a component that is not compliant with some standards, a disassembly work plan can be reactively scheduled. This greatly reduces the risk of discarding non-compliant productions exploiting the reactive reconfigurability of the system.

Within the Industry 4.0 Lab, this kind of activity can be performed by properly modelling the DT tool based on the workstation whose task is to check compliance with quality standards- the camera inspection station. This workstation is placed right after the Robot Assembly cell and checks whether the assembly work done so far is compliant with respect to the work plan requirements. The carrier passes through the workstation, where a camera, together with light beam, checks the conformity. The workstation does not stop the working process if these standards are not satisfied, but it is able to detect it using specific embedded sensors values. The final aim of this station, as it is meant in the framework application, is to be able to reactively schedule a disassembly order that is going to be performed by the robot assembly. The latter, in fact, can be easily configured to perform several activities, both of assembly and of disassembly nature, as previously described.

The steps to effectively run this peculiar simulation model are hereby described:

- A work order that plans the assembly of a front cover with a PCB and one or more fuses is planned and launched.

- $\quad$ The Simulink ${ }^{\circledR}$ MATLAB ${ }^{\circledR}$ function checks at every sampled instant if the $x$ Result variable (sensor that detects if the assembly process done so far is compliant to the work plan; this Boolean variable is true if there is compliance, whilst it is false if not) changes its value from true to false.

- When the order approaches the camera inspection workstation and this condition is verified, the $x$ Result becomes false. This means that the work order that is being assembled is not compliant with the workplan expectations. The case analyzed is relative to the work plan that assembles, inside the covers of the prototypical phone, $\mathrm{PCB}$ and one or more fuses. The xResult changing 
its value to false indicates the lack of fuses on the assembled component, detecting the presence of only the front cover and PCB on the pallet and that at least a fuse is missing from the assembled product. Thus, the simulation code aborts the order that just passed by the camera inspection station thanks to a specific string command sent to the MES.

- As soon as this task is done, a further order with the disassembly work plan is immediately rescheduled on the same pallet, where the front cover with the assembled PCB lies. This work plan has been created and optimized through the first simulation tool described in previous sections.

This kind of work plan is automatically planned by the DT in order to avoid scrapping of material due to the found quality inconsistencies. At this point the whole operation can be considered done and the output changes value. Based on this, thanks to a feedback loop to the Level-2 MATLAB ${ }^{\circledR}$ S-Function, the xResult value is set back to true; otherwise, it would remain false.

The proposed DT, thanks to its high integration level with respect to its physical part given by the MES integration, can be exploited for both the EoL disassembly process and for the scheduling of a new work orders as a reaction to peculiar shop-floor situations. The main objective and benefit that has been proven is the ability of the proposed DT to reactively schedule a disassembly work plan based on certain conditions that can be detected by the embedded sensor system. This outcome is a further validation of the possibility to reconfigure the system and react to shop-floor events that do not block production flow. In fact, the production keeps running, but the lack of compliance with the assembly standards is automatically identified by the DT, and the disassembly work plan is automatically scheduled. According to a broader perspective, this kind of activity allows the possibility to reduce scraps in the facility in which this application is performed. In fact, the pieces that are not compliant with certain standards are usually scrapped, causing potential money loss and inefficiencies for the company. With the use of a DT that is able to perform the activity of disassembly reconfiguration and reactive disassembly, a more sustainable approach can be applied to the manufacturing environment. Accordingly, the materials are not scrapped, but they can be recovered and later re-used according to the application of a CE policy.

\section{Discussion}

The aim of the FENIX project was to demonstrate how the adoption of Circular Economy (CE) principles can enable more sustainable supply chains by increasing the quality, market value, and alternative exploitation of secondary materials. In parallel, a long-lasting European leadership in innovative manufacturing plants engineering will be enabled. Among the FENIX pilots, POLIMI's Industry 4.0 Lab is dedicated to the disassembly of Waste from Electrical and Electronic Equipment (WEEE). This demonstrative, lab-scaled manufacturing process must be adequately reconfigured for managing the selected kind of obsolete products constituting the source of materials to be recovered during FENIX.

Considering the work presented in this paper and developed within the FENIX context, both a VR-based simulation tool and a DT-based simulation tool for assembly and disassembly processes at POLIMI's Industry 4.0 Lab have been tested and optimized, achieving the following summarized results:

1. Reconfiguration of the line (originally designed to perform an assembly process) in order to execute EoL disassembly processes, through virtual design, simulation, and optimization with the $\operatorname{CIROS}^{\circledR}$ software and its uploading on the real system. System reconfigurability at lower levels has been mainly achieved by changing hardware resources (i.e., change of robot tools for disassembly activities), while at the higher levels, it has been mainly achieved by changing software resources (i.e., robot program coding).

2. Creation of a DT of the line, where the data acquired from the field and analyzed in real-time are used to simulate the behavior of the system and allows to evaluate in real-time the energetic performance of the line. This tool allowed possibility for exploiting the IoT for the digitalization 
of CE practices, by implementing smart disassembly process and dynamic feedback control loops [31].

3. Introduction of an energy-based KPI (e-OEE) able to evaluate the energetic performance of the system. This indicator has been introduced in the DT, which is able to extract all the values for real-time energy consumption using an accumulator function. At the end, thanks to the GUI, it is possible to have a clearer and readable way to find useful information for energy management. In this case, CPS and data analytics on energy consumption have been exploited for improving disassembly process performances.

4. Integration of the MES to the DT by using a communication protocol, which is able to give commands to the MES from external sources. With this integration, the monitoring DT became a bi-lateral communication-based DT.

5. Real validation of application models. The disassembly framework concerns the ability to react to a lack of conformity with respect to work orders. When this predicament occurs, the DT is modelled to abort the non-compliant order and re-schedule a disassembly order to avoid scraps.

With the DT, data can be used for different purposes (e.g., applications, analysis, etc.). The OPC-UA communication protocols allows the user to open a gateway to the available data, which is exactly the concept of IoT integration described by the I4.0 paradigm. As presented, the DT is a flexible tool that can be applied both to assembly and disassembly processes without the need of any kind of changes. In this way, the DT allows the evaluation of the behavior of a real system in real-time, enabling the collection of data that can be then used for decision-making process. Through the introduction of an energy-based KPI, and thanks to the exchange of information with the real system in real-time, the platform is able give an overview of the energetic performance of the line in a user-friendly way. The tests reported show how all the data available in the GUI are acquired in order to evaluate the energy consumption of each station of the line in real-time. This is applied to both assembly and disassembly processes with the aim of evaluating their energetic performance.

\section{Conclusions}

Results coming from the literature review show that, even if the intersections between I4.0 technologies and $\mathrm{CE}$ have been assessed by describing the valuable benefits achievable (e.g., optimizing forward and reverse material flows), a real demonstration of these benefits is rarely presented by the experts, especially in the WEEE management field. Furthermore, even if IoT and CPS are described as the most integrated I4.0 technologies able to support the transition toward CE, none of the experts adopted them together with AR/VR and DT simulation tools and robots. The intent of this paper is therefore to present an application case exploiting all these I4.0 technologies together for managing and optimizing a WEEE disassembly process.

Thanks to a new integrated data management along the automation pyramid and thanks to the industrial automation improvements introduced by the fourth industrial revolution, this work proposes an introduction to the following main benefits that I4.0 allows to reach for boosting CE:

- Digitalization of the CE, considering I4.0 technologies as a set of opportunities supporting enterprises in increasing their circular degree through the digital optimization of disassembly processes, increasing their capacity to recover valuable components, and improving materials restoration.

- Process effectiveness, whose goal is not to minimize the flow of materials from the BoL to the EoL but to generate a cyclic metabolism, allowing the materials to maintain their original state, thus being continuously used as input for production systems. This is implemented in this work with the exploitation of VR simulation software applied to advanced manufacturing system for the implementation of disassembly processes.

- CE-related aspects (e.g., resource efficiency and lifecycle management), where I4.0 technologies are enablers of innovative ways for monitoring and optimizing resources performances. The aim is to 
minimize volume and consumption of both energy and material resources. This is implemented in this work by energy data collection and KPI systems creation for decision making process.

The exploitation of these tools and techniques is made possible thanks to the introduction of CPS and I4.0 technologies, making the automation pyramid more flexible and representing the way to create powerful simulation models and a better resource monitoring tool to digitalize CE practice. The intention was to practically demonstrate through a laboratory experiment the incorporation of digital technologies to enable circular industrial metabolism 4.0. The main benefits presented allows for the optimized use of resources for increasing the production cycles sustainability, bringing benefits along the entire product lifecycle. They represent technological boost for the creation of more sustainable and circular business models.

Author Contributions: Conceptualization, R.R., P.R., C.S., L.F., and S.T.; methodology, R.R. and C.S.; formal analysis, R.R. and P.R.; data curation, R.R.; software, R.R.; validation, R.R.; writing-original draft preparation, R.R. and P.R.; writing-review and editing, R.R. and P.R.; visualization, R.R. and P.R.; supervision, L.F. and S.T. All authors have read and agreed to the published version of the manuscript.

Funding: This research was funded by the European Union's Horizon 2020 research and innovation program, grant number 760792. In any case, the present work cannot be considered as an official position of the supporting organization, and it reports only the point of view of the authors.

Conflicts of Interest: The authors declare no conflict of interest.

\section{Abbreviations}

\begin{tabular}{|c|c|}
\hline AR & Augmented Reality \\
\hline BDA & Big Data \& Analytics \\
\hline BoL & Beginning of Life \\
\hline CC & Cloud Computing \\
\hline $\mathrm{CNC}$ & Computer Numerical Control \\
\hline CPS & Cyber-Physical Systems \\
\hline $\mathrm{CE}$ & Circular Economy \\
\hline DES & Discrete Event Simulation \\
\hline DT & Digital Twin \\
\hline e-GUI & energy-Graphical User Interface \\
\hline e-KPI & energy-Key Performance Indicator \\
\hline EoL & End of Life \\
\hline EU & European Union \\
\hline GA & Genetic Algorithm \\
\hline HMI & Human Machine Interface \\
\hline ICT & Information \& Communication Technology \\
\hline IoT & Internet of Things \\
\hline ISA & International Standards of Automation \\
\hline $\mathrm{I} 4.0$ & Industry 4.0 \\
\hline MES & Manufacturing Execution System \\
\hline M2M & Machine-to-Machine \\
\hline OEE & Overall Equipment Effectiveness \\
\hline OPC-UA & OLE for Process Control-Unified Architecture \\
\hline KPI & Key Performance Indicator \\
\hline PCB & Printed Circuit Board \\
\hline PLC & Programmable Logic Controller \\
\hline RFID & Radio-Frequency IDentification \\
\hline SCADA & Supervisory Control And Data Acquisition \\
\hline $\mathrm{SD}$ & System Dynamics \\
\hline VR & Virtual Reality \\
\hline WEEE & Waste from Electrical and Electronic Equipmen \\
\hline
\end{tabular}




\section{References}

1. Cucchiella, F.; D'Adamo, I.; Lenny Koh, S.C.; Rosa, P. Recycling of WEEEs: An economic assessment of present and future e-waste streams. Renew. Sustain. Energy Rev. 2015, 51, 263-272. [CrossRef]

2. Rosa, P.; Terzi, S. Comparison of current practices for a combined management of printed circuit boards from different waste streams. J. Clean. Prod. 2016, 137, 300-312. [CrossRef]

3. Roblek, V.; Meško, M.; Krapež, A. A complex view of industry 4.0. SAGE Open 2016, 6, 2. [CrossRef]

4. Ávila-Gutiérrez, M.J.; Martín-Gómez, A.; Aguayo-González, F.; Córdoba-Roldán, A. Standardization framework for sustainability from circular economy 4.0. Sustainability 2019, 11, 6490. [CrossRef]

5. Rosa, P.; Sassanelli, C.; Urbinati, A.; Chiaroni, D.; Terzi, S. Assessing relations between Circular Economy and Industry 4.0: A systematic literature review. Int. J. Prod. Res. 2019, 58. [CrossRef]

6. Nobre, G.C.; Tavares, E. Scientific literature analysis on big data and internet of things applications on circular economy: A bibliometric study. Scientometrics 2017, 111, 463-492. [CrossRef]

7. Frosch, R.A. Industrial ecology: A philosophical introduction. Proc. Natl. Acad. Sci. USA 1992, 89, 800-803. [CrossRef]

8. Anderberg, S. Industrial metabolism and the linkages between economics, ethics and the environment. Ecol. Econ. 1998, 24, 311-320. [CrossRef]

9. Chertow, M.R. Industrial symbiosis: Literature and taxonomy. Annu. Rev. Energy Environ. 2000, 25, 313-337. [CrossRef]

10. Yuan, Z.; Bi, J.; Moriguchi, Y. The circular economy: A new development strategy in China. J. Ind. Ecol. 2006, 10, 4-8. [CrossRef]

11. Florin, N.; Madden, B.; Sharpe, S.; Benn, S.; Agarwal, R.; Perey, R.; Giurco, D. Shifting Business Models for a Circular Economy: Metals Management for Multi-Product-Use Cycles; UTS: Sydney, Australia, 2015.

12. The Ellen MacArthur Foundation. Towards a Circular Economy: Business Rationale for an Accelerated Transition; The Ellen MacArthur Foundation: Cowes, UK, 11 March $2015 . \quad$ Available online: https://www.ellenmacarthurfoundation.org/assets/downloads/TCE_Ellen-MacArthur-Foundation_9Dec-2015.pdf (accessed on 11 March 2020).

13. MacArthur, E. Circularity Indicators: An Approach to Measuring Circularity. Methodology 2015. [CrossRef]

14. ANSI/ISA. Enterprise-Control System Integration-Part 1: Models and Terminology; American National Standards Institute: Washington, DC, USA, 2010.

15. Pathfinder project. Pointing out research priorities leading to the next generation of simulation and forecasting technologies. 2014. Available online: https://pathfinderproject.eu/downloads/results/Pathfinder_ WhitePaper1.pdf (accessed on 11 March 2020).

16. Negri, E.; Fumagalli, L.; Macchi, M. A review of the roles of digital twin in CPS-based production systems. Procedia Manuf. 2017, 11, 939-948. [CrossRef]

17. Fumagalli, L.; Pala, S.; Garetti, M.; Negri, E. Ontology-Based Modeling of Manufacturing and Logistics Systems for a New MES Architecture; Springer: Berlin/Heidelberg, Germany, 2014; pp. 192-200.

18. Yang, S.; Raghavendra, M.R.A.; Kaminski, J.; Pepin, H. Opportunities for industry 4.0 to support remanufacturing. Appl. Sci. 2018, 8, 7. [CrossRef]

19. Lee, J.; Bagheri, B.; Kao, H.-A. A cyber-physical systems architecture for industry 4.0-based manufacturing systems. Manuf. Lett. 2015, 3, 18-23. [CrossRef]

20. Jazdi, N. Cyber physical systems in the context of industry 4.0. In Proceedings of the 2014 IEEE International Conference on Automation, Quality and Testing, Robotics, Cluj-Napoca, Romania, 22-24 May 2014. [CrossRef]

21. Baheti, R.; Gill, H. Cyber-physical systems. Impact Control Technol. 2011, 12, 161-166.

22. Stock, T.; Seliger, G. Opportunities of sustainable manufacturing in industry 4.0. Procedia CIRP 2016, 40, 536-541. [CrossRef]

23. Jabbour, A.B.L.D.S.; Jabbour, C.J.C.; Filho, M.G.; Roubaud, D. Industry 4.0 and the circular economy: A proposed research agenda and original roadmap for sustainable operations. Ann. Oper. Resour. 2018, 270, 273-286.

24. Bressanelli, G.; Adrodegari, F.; Perona, M.; Saccani, N. Exploring how usage-focused business models enable circular economy through digital technologies. Sustainability 2018, 10, 639. [CrossRef]

25. Pagoropoulos, A.; Pigosso, D.C.A.; McAloone, T.C. The emergent role of digital technologies in the circular economy: A review. Procedia CIRP 2017, 64, 19-24. [CrossRef] 
26. Davis, J.; Edgar, T.; Porter, J.; Bernaden, J.; Sarli, M. Smart manufacturing, manufacturing intelligence and demand-dynamic performance. Comput. Chem. Eng. 2012, 47, 145-156. [CrossRef]

27. Lee, J.; Kao, H.; Yang, S. Service innovation and smart analytics for Industry 4.0 and big data environment. Procedia CIRP 2014, 16, 3-8. [CrossRef]

28. De Man, J.C.; Strandhagen, J.O. An industry 4.0 research agenda for sustainable business models. Procedia CIRP 2017, 63, 721-726. [CrossRef]

29. Chang, M.M.L.; Ong, S.K.; Nee, A.Y.C. Approaches and challenges in product disassembly planning for sustainability. Procedia CIRP 2017, 60, 506-511. [CrossRef]

30. Hatzivasilis, G.; Fysarakis, K.; Soultatos, O.; Askoxylakis, I.; Papaefstathiou, I.; Demetriou, G. The industrial Internet of things as an enabler for a circular economy Hy-LP: A novel IIoT protocol, evaluated on a wind park's SDN/NFV-enabled 5G industrial network. Comput. Commun. 2018, 119, 127-137. [CrossRef]

31. Reuter, M.A. Digitalizing the circular economy: Circular economy engineering defined by the metallurgical internet of things. Metall. Mater. Trans. B Process Metall. Mater. Process. Sci. 2016, 47, 3194-3220. [CrossRef]

32. Weyer, S.; Meyer, T.; Ohmer, M.; Gorecky, D.; Zühlke, D. Future modeling and simulation of CPS-based factories: An example from the automotive industry. IFAC PapersOnLine 2016, 49, 97-102. [CrossRef]

33. Legat, C.; Seitz, C.; Lamparter, S.; Feldmann, S. Semantics to the shop floor: Towards ontology modularization and reuse in the automation domain. IFAC Proc. Vol. 2014, 47, 3444-3449. [CrossRef]

34. Negri, E.; Fumagalli, L.; Garetti, M.; Tanca, L. Computers in industry requirements and languages for the semantic representation of manufacturing systems. Comput. Ind. 2016, 81, 55-66. [CrossRef]

35. Posada, J.; Toro, C.; Barandiaran, I.; Oyarzun, D.; Stricker, D.; de Amicis, R.; Pinto, E.B.; Eisert, P.; Döllner, J.; Vallarino, I. Visual computing as a key enabling technology for industrie 4.0 and industrial internet. IEEE Comput. Graph. Appl. 2015, 35, 26-40. [CrossRef]

36. Cimino, C.; Negri, E.; Fumagalli, L. Review of digital twin applications in manufacturing. Comput. Ind. 2019, 113, 103130. [CrossRef]

37. Wang, X.V.; Wang, L. Digital twin-based WEEE recycling, recovery and remanufacturing in the background of Industry 4.0. Int. J. Prod. Res. 2019, 57, 3892-3902. [CrossRef]

38. Merdan, M.; Lepuschitz, W.; Meurer, T.; Vincze, M. Towards ontology-based automated disassembly systems. In Proceedings of the IECON 2010-36th Annual Conference on IEEE Industrial Electronics Societyp, Glendale, AZ, USA, 7-10 November 2010. [CrossRef]

39. PricewaterhouseCoopers (PWC), Making business and economic sense of climate change: Pwc sustainability and climate change blog. Available online: https://www.pwc.com/gx/en/services/sustainability.html. (accessed on 13 March 2020).

40. French, R.; Benakis, M.; Marin-Reyes, H. Intelligent sensing for robotic re-manufacturing in aerospace-An industry 4.0 design based prototype. In Proceedings of the IRIS 2017-5th IEEE International Symposium on Robotics and Intelligent Sensors, Ottawa, ON, Canada, 5-7 October 2017; pp. 272-277. [CrossRef]

41. Barbosa, J.; Leitão, P.; Trentesaux, D.; Colombo, A.W.; Karnouskos, S. Cross benefits from cyber-physical systems and intelligent products for future smart industries. In Proceedings of the INDIN 2016-14th IEEE International Conference on Industrial Informatics, Poitiers, France, 19-21 July 2016; pp. 504-509. [CrossRef]

42. Hehenberger, P.; Vogel-Heuser, B.; Bradley, D.; Eynard, B.; Tomiyama, T.; Achiche, S. Design, modelling, simulation and integration of cyber physical systems: Methods and applications. Comput. Ind. 2016, 82, 273-289. [CrossRef]

43. Schroeder, G.N.; Steinmetz, C.; Pereira, C.E.; Espindola, D.B. Digital twin data modeling with automationml and a communication methodology for data exchange. IFAC PapersOnLine 2016, 49, 12-17. [CrossRef]

44. Schroeder, G.N.; Rodrigues, R.; Espindola, D.; Garcia, N.; Muller, I. Visualising the digital twin using web services and augmented reality. In Proceedings of the INDIN' 16-14th IEEE International Conference on Industrial Informatics, Poitiers, France, 19-21 July 2016; pp. 522-527. [CrossRef]

45. D'Adamo, I.; Ferella, F.; Gastaldi, M.; Maggiore, F.; Rosa, P.; Terzi, S. Towards sustainable recycling processes: Wasted printed circuit boards as a source of economic opportunities. Resour. Conserv. Recycl. 2019, 149, 455-467. [CrossRef]

46. Ji, L.; Liu, C.; Huang, L.; Huang, G. The evolution of Resources Conservation and Recycling over the past 30 years: A bibliometric overview. Resour. Conserv. Recycl. 2018, 134, 34-43. [CrossRef]

47. Goodall, P.A.; Sharpe, R.G.; West, A.A. A data-driven simulation to support remanufacturing operations. Comput. Ind. 2019, 105, 48-60. [CrossRef] 
48. Alqahtani, A.Y.; Gupta, S.M.; Nakashima, K. Warranty and maintenance analysis of sensor embedded products using internet of things in industry 4.0. Int. J. Prod. Econ. 2019, 208, 483-499. [CrossRef]

49. Joshi, A.D.; Gupta, S.M. Evaluation of design alternatives of End-Of-Life products using internet of things. Int. J. Prod. Econ. 2019, 208, 281-293. [CrossRef]

50. Marconi, M.; Germani, M.; Mandolini, M.; Favi, C. Applying data mining technique to disassembly sequence planning: A method to assess effective disassembly time of industrial products applying data mining technique to disassembly sequence planning: A method to assess effective disassembly time of industry. Int. J. Prod. Res. 2018, 57, 599-623. [CrossRef]

51. Sharpe, R.G.; Goodall, P.A.; Neal, A.D.; Conway, P.P.; West, A.A. Cyber-physical systems in the re-use, refurbishment and recycling of used electrical and electronic equipment. J. Clean. Prod. 2018, 170, 351-361. [CrossRef]

52. Kuik, S.S.; Kaihara, T.; Fujii, N. Product recovery configuration decisions for achieving sustainable manufacturing. Procedia CIRP 2016, 41, 258-263. [CrossRef]

53. Rönnlund, I.; Reuter, M.; Horn, S.; Aho, J.; Aho, M.; Päällysaho, M.; Ylimäki, L.; Pursula, T. Eco-efficiency indicator framework implemented in the metallurgical industry: Part 1-A comprehensive view and benchmark. Int. J. Life Cycle Assess. 2016, 21, 1473-1500. [CrossRef]

54. Smith, J.S. Survey on the use of simulation for manufacturing system design and operation. J. Manuf. Syst. 2013, 22, 157-171. [CrossRef]

55. Negahban, A.; Smith, J.S. Simulation for manufacturing system design and operation: Literature review and analysis. J. Manuf. Syst. 2014, 33, 241-261. [CrossRef]

56. Mourtzis, D.; Doukas, M.; Bernidaki, D. Simulation in manufacturing: Review and challenges. Procedia CIRP 2014, 25, 213-229. [CrossRef]

57. Taraman, S.R.; Lee, D.G. Manufacturing Lines Design and Analysis Utilizing Systems Simulation-A Case Study. In Proceedings of the 1983 ASME International Computers In Engineering Conference, Chicago, IL, USA, August 1983; pp. 99-105.

58. Festo ${ }^{\circledR}$ Didactics-Festo SE \& Co. KG, Ruiter Straße 82, 73734, Esslingen, Germany. Available online: https://www.festo.com/cms/it_it/index.htm (accessed on 13 March 2020).

59. Festo ${ }^{\circledR}$ Didactics-CIROS software. 2018. Available online: https://www.festo-didactic.com/int-en/learningsystems/software-e-learning/ciros/?fbid=aW50LmVuLjU1Ny4xNy4yMC4xMTEw (accessed on 13 March 2020).

60. Rosen, R.; Von Wichert, G.; Lo, G.; Bettenhausen, K.D. About the importance of autonomy and digital twins for the future of manufacturing. ScienceDirect 2015, 48, 567-572. [CrossRef]

61. Colombo, A.W.; Bangemann, T.; Stluka, P.; Harrison, R.; Jammes, F. Industrial cloud-based cyber-physical systems. Imc Aesop Approach 2014, 22, 4-5.

62. Caggiano, A. Cloud-based manufacturing process monitoring for smart diagnosis services. Int. J. Comput. Integr. Manuf. 2018, 31, 612-623. [CrossRef]

63. Hedman, R.; Subramaniyan, M.; Almström, P. Analysis of critical factors for automatic measurement of OEE. Procedia CIRP 2016, 57, 128-133. [CrossRef]

64. Eguia, I.; Lozano, S.; Racero, J.; Guerrero, F. A methodological approach for designing and sequencing product families in reconfigurable disassembly systems. J. Ind. Eng. Manag. 2011, 4, 418-435. [CrossRef]

65. Bi, Z.M.; Lang, S.Y.T.; Shen, W.; Wang, L. Reconfigurable manufacturing systems: The state of the art. Int. J. Prod. Res. 2008, 46, 967-992. [CrossRef]

66. MATLAB ${ }^{\circledR}$, “MATLAB ${ }^{\circledR}$ Library-TCP/IP Function. Available online: https://it.mathworks.com/help/ instrument/tcpip.html (accessed on 11 March 2020).

(C) 2020 by the authors. Licensee MDPI, Basel, Switzerland. This article is an open access article distributed under the terms and conditions of the Creative Commons Attribution (CC BY) license (http://creativecommons.org/licenses/by/4.0/). 